\title{
Rescoring of docking poses using force field-based methods
}

\author{
Nina M Fischer", WM Schneider, O Kohlbacher \\ From 5th German Conference on Cheminformatics: 23. CIC-Workshop \\ Goslar, Germany. 8-10 November 2009
}

Existing protein-ligand docking methods computationally screen thousands to millions of organic molecules against protein structures, trying to find those with complementary shapes and highest binding free energies. To allow large molecular databases to be screened rapidly, simple and approximative scoring functions are used as a fast filter, resulting in low hit rates. Therefore, docking hit lists are commonly rescored in a final step by more rigorous and time-consuming methods to gain a more accurate final list of ranked compounds.

Molecular mechanics Poisson-Boltzmann surface area (MM-PBSA) or generalized Born surface area (MMGBSA) methods are currently considered to be suitable techniques for rescoring. These physically realistic approaches incorporate more sophisticated models for solvation and electrostatic interactions than most scoring functions. Hence, they can discriminate more reliably between correct and incorrect docking poses.

A high-throughput rescoring protocol using force field-based methods has been proposed by Brown and Muchmore [1]. They used 18 in-house urokinase-ligand crystal structures and their corresponding experimentally determined binding free energies as a test set. On the basis of this rescoring protocol we tested several molecular dynamics simulation protocols in combination with different MM-PBSA, and MM-GBSA calculation procedures using Amber 10 [2] and Gromacs 4 [3]. Considering performance and accuracy, our best rescoring protocol performs similarly to the one described by Brown and Muchmore [1]. It has a comparable run-time and achieves a correlation between experimental and rescored values of 0.88 compared to 0.87 .

However, we used urokinase-ligand complexes generated using the docking program Glide [4] instead of crystal structures. Additionally, this shows that our

Eberhard Karls University, Center for Bioinformatics Tübingen, Sand 14, 72076 Tübingen, Germany rescoring protocol improves the correlation of 0.57 between experimental values and Glide scores significantly to 0.88 , thereby achieving a more accurate list of ranked compounds.

The protocol will be incorporated into BALLView [5], an open-source molecular viewer and modeling tool. Thus, it will be available free of charge and can be conveniently used to rescore (docked) protein-ligand complexes.

\section{Published: 4 May 2010}

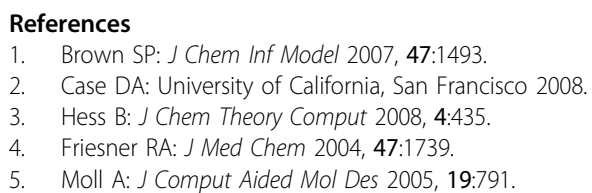

doi:10.1186/1758-2946-2-S1-P52

Cite this article as: Fischer et al:: Rescoring of docking poses using force field-based methods. Journal of Cheminformatics 2010 2(Suppl 1):P52. 